\title{
A Resource Allocation Scheme with Delay Optimization Considering mmWave Wireless Networks
}

\author{
Marcus V. G. Ferreira1, Flávio H. T. Vieira1,2, Marcos N. L. Carvalho ${ }^{2}$ \\ ${ }^{1}$ Instituto de Informática, Universidade Federal de Goiás, Goiânia, Goiás, Brasil \\ ${ }^{2}$ Escola de Engenharia Elétrica, Mecânica e de Computação, Universidade Federal de Goiás, Goiânia, Goiás, Brasil \\ Email: marcusferreira@inf.ufg.br, flavio_vieira@ufg.br, marcosnlc4@gmail.com
}

How to cite this paper: Ferreira, M.V.G., Vieira, F.H.T. and Carvalho, M.N.L. (2020) A Resource Allocation Scheme with Delay Optimization Considering mmWave Wireless Networks. Int. J. Communications, Network and System Sciences, 13, 105-119. https://doi.org/10.4236/ijcns.2020.137007

Received: July 5, 2020

Accepted: July 28, 2020

Published: July 31, 2020

Copyright $\odot 2020$ by author(s) and Scientific Research Publishing Inc. This work is licensed under the Creative Commons Attribution International License (CC BY 4.0).

http://creativecommons.org/licenses/by/4.0/

(c) (i) Open Access

\begin{abstract}
This paper presents a resource allocation scheme for wireless networks, aiming at optimizing the users' data delay. It is proposed to provide optimal delay allocation by solving an optimization problem using idle state prediction and considering 5G characteristics such as mmWave propagation. The performance of the resource allocation algorithm is verified and compared with others from the literature using computational simulations in terms of Quality of Service $(\mathrm{QoS})$ parameters such as throughput, delay, fairness index, loss rate and computational complexity. In these simulations, it is also considered the mmWave propagation and carrier aggregation technology for wireless next generation systems, in order to verify the system performance in a high data rate scenario.
\end{abstract}

\section{Keywords}

Delay, QoS, mmWave, Carrier Aggregation, 5G

\section{Introduction}

Aiming to support next-generation wireless networks, we address in this paper a scenario with millimeter waves (mmWaves) above $6 \mathrm{GHz}$ and a radio frame structure with spacing between sub-carriers of $120 \mathrm{kHz}$. The $5 \mathrm{G}$ specifications of Release 15 of the 3rd Generation Partnership Project (3GPP) standardization organization were followed, in order to verify the performance of the algorithm in a current simulation scenario with a high transmission rate [1] [2] [3].

In this work, it is also considered in the simulations, techniques such as 256-QAM (Quadrature Amplitude Modulation) and carrier aggregation. These 
technologies are introduced in Long-Term Evolution - Advanced (LTE-A) networks and developed by operators to promote higher data rates, better coverage, and lower latency [4].

There are many proposals for resource allocation schemes in wireless networks [5] [6] [7]. LIN SU et al. (2012) [7] proposed a coding scheme based on the Particle Swarm Optimization (PSO) heuristic to map particle solutions and find an operation to discretize the position and velocity of particles and then establish a fitness function to handle constraints through a penalty function. GUAN et al. [6] proposed an algorithm that aims to guarantee the minimum transmission rate criteria required by the user. This algorithm, called Quality of Service (QoS) guaranteed in this paper, first estimates the number of blocks required for each user and then allocates these blocks to users according to their priorities. FERREIRA et al. (2015) [5] presented an algorithm, namely Min-delay algorithm, that considers estimated system delay values, channel quality and the maximum delay value for each user in order to decide on the scaling of available resources. However, these algorithms do not apply optimization methods to directly minimize system delay for users as our proposal does.

In the mentioned context of resource allocation, the delay is considered essential, especially for real-time applications with variable transfer rates and specific band requirements such as the Voice over Internet Protocol (VoIP) and videoconference services. It is also important to highlight the need to develop new techniques to facilitate the rigorous QoS requirements for $5 \mathrm{G}$ networks in terms of delays to be met [8].

The present paper proposes a resource block allocation scheme for wireless networks considering current and next-generation techniques, aiming at optimizing the system delay and obtaining values for other QoS parameters compatible with those of other schedulers from the literature. In this work, it is proposed to provide optimal delay allocation for networks with 5G characteristics by solving an optimization problem using idle state prediction and by considering carrier aggregation and mmWave propagation. Comparisons are made with other algorithms in the literature through statistical data in terms of QoS parameters, proving the efficiency of the proposed algorithm.

\section{Transmission System Model}

Consider a wireless network with only one evolved Node B (eNB), consisting of $N$ items of User Equipment (UE) such as $\alpha=\left\{\alpha_{1}, \alpha_{2}, \cdots, \alpha_{N}\right\}$. Each $\alpha_{n} \in \alpha$ user can have different Carrier Aggregation (CA) capacities, which are modeled as $\mu=\left\{\mu_{n} \mid \mu_{n} \in\{1,2, \cdots, 32\}\right\}_{1 \times N}$, where $\mu_{n}$ represents the maximum number of Component Carriers (CC) that can be supported by $\alpha_{n} \in \alpha$.

All UEs in a given Transmission Time Interval (TTI) compete for $M$ orthogonal CCs without overlapping, represented by $\beta=\left\{\beta_{1}, \beta_{2}, \cdots, \beta_{M}\right\}$ where each $\beta_{m} \in \beta$ has a different number of Resource Blocks (RBs) $\gamma_{m}$.

Assuming that the maximum Modulation and Coding Scheme (MCS) index of all UEs in different RBs is modeled as in [4]; that is: 


$$
K=\left\{k_{m, p, n} \mid k_{m, p, n} \in\{0,1, \cdots, h\}\right\}_{M \times P \times N},
$$

where $k_{m, p, n}$ represents the maximum MCS index for $\alpha_{n}$ in $\beta_{m}$ in the RB $p$. The value of $k_{m, p, n}$ depends on the channel quality interval between 0 and $h$. In turn, the uplink value of $h$ is 22 , its downlink value is 28 , and $P=\max \gamma$. A resource block allocation matrix is also defined as a binary matrix [4]:

$$
A=\left\{a_{m, p, n} \mid a_{m, p, n} \in\{0,1\}\right\}_{M \times P \times N},
$$

representing the $\mathrm{RB}$ allocation map where $a_{m, p, n}=1$ if and only if the $\mathrm{RB} p$ using CC $\beta_{m}$ is allocated only for $\alpha_{n}$ and $a_{m, p, n}=0$ otherwise. The resource allocation matrix $A$ should meet the interference requirement defined in equation (3), i.e., two or more UEs cannot use the same RB simultaneously [4]:

$$
\sum_{n=1}^{N} a_{m, p, n} \leq 1 \text { for } 1 \leq p \leq P, 1 \leq m \leq M .
$$

A CC allocation matrix that defines which resource blocks are allocated to which UEs is represented as a binary matrix $M \times N$ relative to the total number of CCs $M$ and the number of UEs $N$, given by [4]:

$$
E=\left\{e_{m, n} \mid e_{m, n}=1 \leftrightarrow \sum_{p=1}^{P} a_{m, p, n} \geq 1\right\}_{M \times N},
$$

where $e_{m, n}$ represents the condition if $\beta_{m}$ is attributed or not to $\alpha_{n}$. To represent the MCS allocated for $\alpha_{n}$ in a certain TTI, a user matrix is defined as $[4]:$

$$
B=\left\{b_{m, n} \mid b_{m, n} \in\{0,1, \cdots, h\}\right\}_{M \times N},
$$

where $b_{m, n}$ represents the MCS index allocated for $\alpha_{n}$ in $\beta_{m}$ for each TTI. The 3GPP specifies in [9] the transfer rate that corresponds to each MCS index.

In this work, to represent the relationship between the MCS index and the transfer rate, the notation $r=R(b)$ is used, where $R$ scans each MCS index for each transfer rate according [9]. In other words, $r$ is the transfer rate obtained for each UE in an RB with MCS $b$. Therefore, the user transfer rate is defined as $R=\left\{r_{m, n} \mid r_{m, n}=R\left(b_{m, n}\right)\right\}_{M \times N}$, i.e., an $M \times N$ matrix where $r_{m, n}$ presents the transfer rate per RB for $\alpha_{n}$ in $\beta_{m}$.

The eNB is responsible for the entire admission procedure, resource scheduling, and link adaptation. After receiving the Channel State Information (CSI) of all UEs, a resource allocation map is built, and the MCS index is determined by the eNB and then sent to each UE through the control channels. According to the MCS index, the modulation type and the encoding rate for each UE in each attributed CC can be determined. The 3GPP specifies in [9] the transfer rate that corresponds to each MCS index.

Many allocation schemes are based on the maximization of the total system throughput with the constraint of the minimum transfer rate required per user. In this case, the optimization objective function is defined as the throughput provided in a given TTI $t$, and can be calculated as: 


$$
f^{(t)}=\sum_{m=1}^{M} \sum_{p=1}^{P} \sum_{n=1}^{N} r_{m, n}^{(t)} \times a_{m, p, n}^{(t)},
$$

where $r_{m, n}^{(t)}$ and $a_{m, p, n}^{(t)}$ are $r_{m, n}$ and $a_{m, p, n}$ in the TTI $t$, respectively. This is subject to:

$$
\begin{gathered}
\sum_{n=1}^{N} a_{m, p, n} \leq 1, \\
\sum_{m=1}^{M} e_{m, n} \leq \mu_{n}, \\
b_{m, n} \leq k_{m, p, n}, \\
r_{m, n} \geq r_{m, n}^{\min },
\end{gathered}
$$

for $1 \leq p \leq P, 1 \leq m \leq M$, and $1 \leq n \leq N$, where $e_{m, n}$ represents the condition if $\beta_{m}$ is attributed or not to $\alpha_{n}$ and $b_{m, n}$ represents the MCS index allocated for $\alpha_{n}$ in $\beta_{m}$ for each TTI.

Equation (7) ensures that each RB in the network is attributed to one UE at most. Equation (8) guarantees that the number of CCs attributed to each UE is smaller than its aggregation capacity. Equation (9) guarantees that the MCS index for each UE in each CC is lower than the maximum MCS index supported by each RB attributed to its corresponding CC. Equation (10) guarantees that the schedulers meet the minimum required transfer rate for each user.

\section{Resource Allocation with Delay Optimization}

Consider at first the optimization problem for a static channel $h$ and the initial status of buffer $q(t=1)$. The mean arrival rate is denoted as $\bar{a}$, and it is assumed that no packages arrive after $t=0$. Then, the size of the observation window $T$ is selected with $q_{n}(T)=0, \forall n \in N$ so that the buffers are completely unoccupied in the time window. In this work, it is proposed to obtain an optimal delay scheduling scheme by solving the following optimization problem:

$$
\min \sum_{t=1}^{T}\left(\sum_{n=1}^{N} \frac{q_{n}^{1}}{\bar{a}_{n}}-\sum_{n=1}^{N}(T-t) \frac{r_{n}^{t}}{\bar{a}_{n}}\right)
$$

subject to:

$$
\begin{gathered}
r^{t} \in C, \\
q_{n}^{1}-\sum_{u=1}^{t} r_{n}^{u} \geq 0, \forall n \in N, t \in[1, \cdots, T],
\end{gathered}
$$

where $q_{n}^{t}$ and $r_{n}^{t}$ denote the size of the queue and the transfer rate of the user $n$ at time $t$, and $C$ denotes the instantaneous capacity region of the system.

The downlink maximum data rate for an UE transmission in Cyclic Prefix Orthogonal Frequency Division Multiplexing (CP-OFDM) wireless system with carrier aggregation is given by [10]:

$$
C_{\max }=10^{-6} \cdot \sum_{j=1}^{J}\left(v_{\text {Layers }}^{(j)} \cdot Q_{m}^{(j)} \cdot f^{(j)} \cdot R_{\max } \cdot \frac{N_{P R B}^{B W(j), v} \cdot 12}{T_{s}^{v}} \cdot\left(1-O H^{(j)}\right)\right),
$$


where $J$ is the number of aggregated component carriers, $v_{\text {Layers }}^{(j)}$ is the maximum number of supported layers, $Q_{m}^{(j)}$ is the maximum supported modulation order, $f^{(j)}$ is the scaling factor, $v$ is the numerology, $T_{s}^{v}$ is the average Cyclic Prefix - Orthogonal Frequency Division Multiplexing (CP-OFDM) symbol duration in a subframe for numerology $v, N_{P R B}^{B W(j), v}$ is the maximum RB allocation in bandwidth $B W^{(j)}$ and $O H^{(j)}$ is the system overhead.

Applying Lagrangian function and denoting $\eta_{n}^{*}=T-\bar{a}_{n} \sum_{u=1}^{T} \lambda_{n}^{u}$, the optimal $\mu_{n}^{t}$ is found [11]:

$$
\mu_{n}^{t}= \begin{cases}\frac{\eta_{n}^{*}-t+1}{\bar{a}_{n}}, & n \leq \eta^{*} \\ 0, & n>\eta^{*}\end{cases}
$$

and the delay optimization problem for a CP-OFDM wireless system with carrier aggregation becomes:

$$
\max \sum_{t=1}^{T} \sum_{n=1}^{N} \mu^{t \mathrm{~T}} \cdot r
$$

subject to the following constraints:

$$
\begin{gathered}
r^{t} \in C, \\
C \leq 10^{-6} \cdot \sum_{j=1}^{J}\left(v_{\text {Layers }}^{(j)} \cdot Q_{m}^{(j)} \cdot f^{(j)} \cdot R_{\max } \cdot \frac{N_{P R B}^{B W(j), v} \cdot 12}{T_{s}^{v}} \cdot\left(1-O H^{(j)}\right)\right) .
\end{gathered}
$$

We propose Algorithm 1 to solve the optimization problem represented by Equations (16), (17) and (18), by estimating the queue status via estimation of the non idle state parameter. The parameter $\eta_{n}^{*}$ in Equation (15) can be obtained by the iterative approximation described in Algorithm 1 presented bellow, where $\lceil\eta\rceil$ denotes a smaller integer larger than $\eta$, and $\varepsilon$ is the predefined tolerable error of $\eta$. In the system, with the new arrival of packages, the weight vector $\tilde{\mu}^{1}$ should be recalculated according to the new queue status. The allocation rate is determined with $\tilde{\mu}^{1}$ and the current state of channel $h$.

It is important to highlight that Algorithm 1 can also be used if the state of channel $h$ varies over time and the base station has anticipated information on the state of each channel. In this work, it is assumed that the base station only has information of the current channel state. Additionally, the process of package arrival is not ergodic and it is difficult to predict. To avoid a possible infinite queue delay, it is required that the queue state be maintained in a stable state for each expected arrival rate $\rho$ within the ergodic capacity region. Thus, the optimization problem (throughput maximization with constraints) can be solved using Algorithm 1 once it is intended to find the optimal values for $r$ that attain the capacity region given by Equation (18).

In other words, this work proposes a scheduling algorithm that is Algorithm 1, for resource allocation of wireless networks that is based on Equation (15) for estimating optimal rate values that should be allocated to users, considering aggregation of subcarriers and Cyclic Prefix - Orthogonal Frequency Division Multiplexing (CP-OFDM) waveform. The use of these techniques influences the 


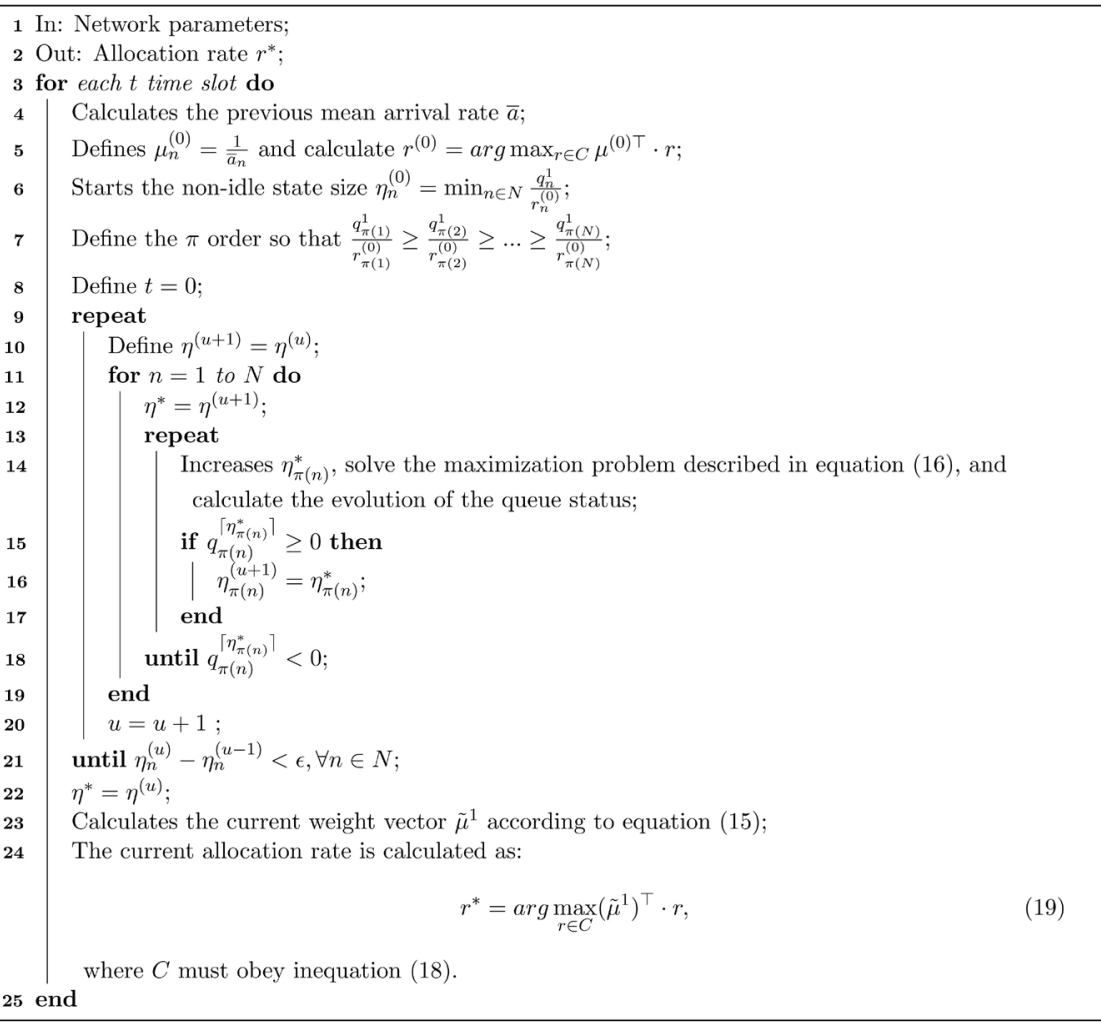

Algorithm 1. Proposed algorithm: Scheduling for delay optimization.

capacity of the channel in Algorithm 1 and consequently the final transfer rate for the users. Hence, the purpose is to improve the network performance, mainly in terms of the users' data delay and throughput.

The proposed resource allocation algorithm adds to the optimization process a part dedicated to the minimization of the delay based on the behavior of the queue size in the user buffer in order to maximize throughput. In fact, the other algorithms considered in this study aim at maximizing the total network throughput; however, they do not consider the prediction of idle state.

\section{Simulations and Results}

This section presents the results of simulations of the proposed resource block allocation algorithm in comparison with other algorithms from the literature, implemented in MATLAB version R2018a. The simulation considered the same wireless network scenario for all of the algorithms with subcarrier aggregation and CP-OFDM modulation [12], in addition to the same channel modeling and traffic conditions.

The downlink transfer scenario and the channel conditions for each user and RBs were generated for each TTI according to the parameters presented in Table 1. The considered values for these parameters are similar to those found in references [1] [2] [5] [6] [7] [13]. It is considered the Tapped Delay Line - A (TDL-A) multipath model [1], suitable for the mmWave scenario, and the Rayleigh fading, to verify the channel modeling impacts via simulations. The bit rate 
Table 1. Simulation parameters for downlink transfer scenario and channel modeling.

\begin{tabular}{|c|c|}
\hline Bandwidth (MHz) & $3 \times 396$ \\
\hline Subcarrier spacing $(\mathrm{KHz})$ & 120 \\
\hline Number of resource blocks per & 275 \\
\hline \multicolumn{2}{|l|}{ component } \\
\hline Carrier frequency $(\mathrm{GHz})$ & 26 \\
\hline Minimum transfer rate & Traffic average \\
\hline Number of simulated TTIs & 1000 \\
\hline Waveform & CP-OFDM \\
\hline \multirow[t]{2}{*}{ Modulation } & QPSK, 16-QAM, 64-QAM and \\
\hline & 256-QAM \\
\hline \multirow[t]{3}{*}{ Multipath model } & TDL-A Urban Macro Non \\
\hline & Line of Sight (NLOS) and Rayleigh \\
\hline & Extended Typical Urban (ETU) \\
\hline Log normal shadowing (dB) & Mean 0 and standard deviation 7.8 \\
\hline Distance from UE to eNB (km) & 0.5 \\
\hline White-noise power density & -174 \\
\hline \multicolumn{2}{|l|}{$(\mathrm{dBm} / \mathrm{Hz})$} \\
\hline Maximum eNB transfer power & 35 \\
\hline \multicolumn{2}{|l|}{$(\mathrm{dBm})$} \\
\hline eNB height (m) & 25 \\
\hline eNB antenna gain after cable & 15 \\
\hline \multicolumn{2}{|l|}{ loss $(\mathrm{dBi})$} \\
\hline UE antenna gain $(\mathrm{dBi})$ & 0 \\
\hline UE noise figure $(\mathrm{dB})$ & 9 \\
\hline UE interference margin $(\mathrm{dB})$ & 4 \\
\hline UE speed $(\mathrm{km} / \mathrm{h})$ & 3 \\
\hline
\end{tabular}

and MCS index associated with the Signal-plus-Noise Ratio (SNR) are determined using a 4-bit Channel Quality Indicator (CQI) based mapping according to [9] (Figure 1 and Figure 2).

The simulations were carried out to compare the results of the proposed algorithm with the following resource allocation algorithms (briefly described in the Introduction): PSO-based [7], QoS guaranteed [6] and delay minimization, named the Min-delay [5]. In Figure 3, Figure 7 and Figure 9, the results correspond to the average of 1000 algorithm runs for being statistically significative in describing average behavior.

Figure 3 and Figure 4 indicates that the proposed algorithm exhibited better performance in terms of total throughput for both scenarios with different channel modeling types. This behavior occurs because the proposed algorithm 


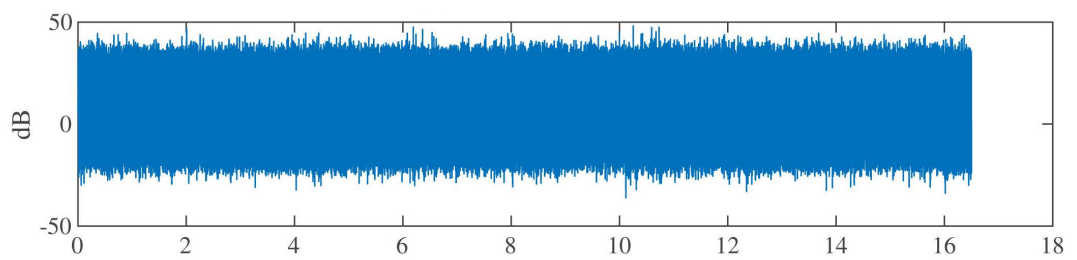

(a)

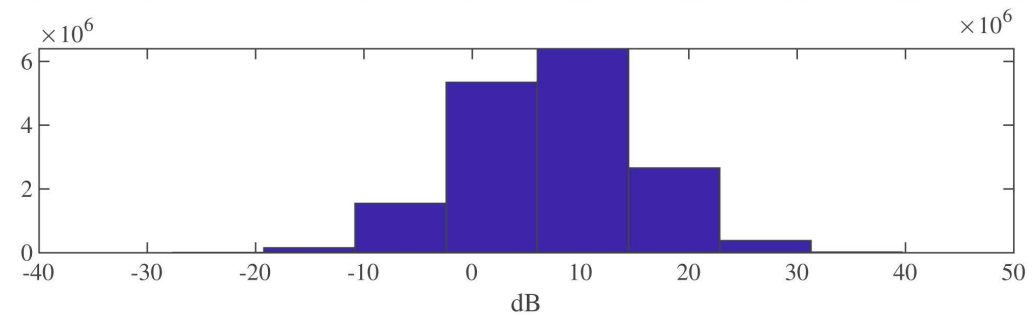

(b)

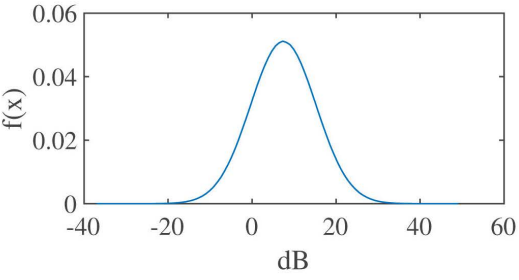

(c)

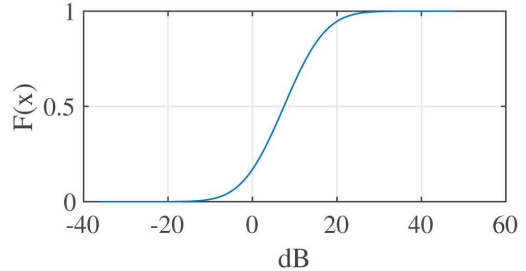

(d)

Figure 1. TDL-A channel modeling statistics. (a) SINR statistics TDL-A; (b) Histogram; (c) Estimated PDF; (d) Empirical CDF.

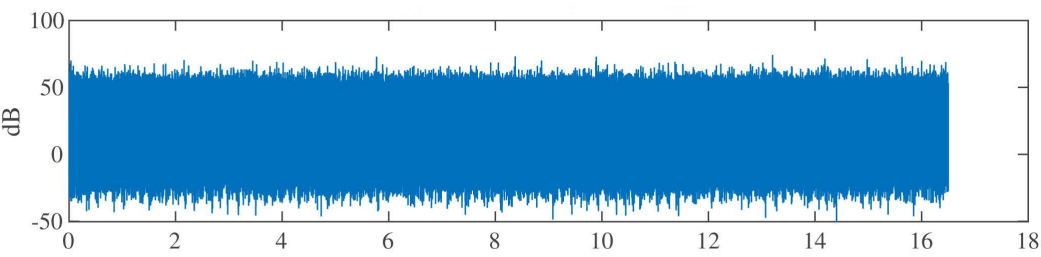

(a)

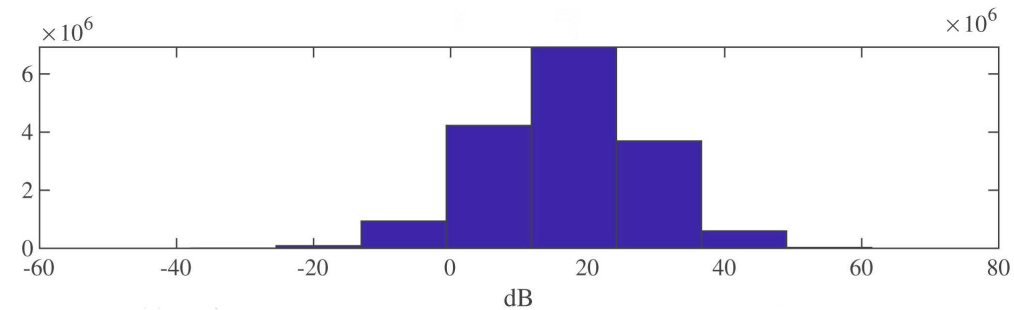

(b)

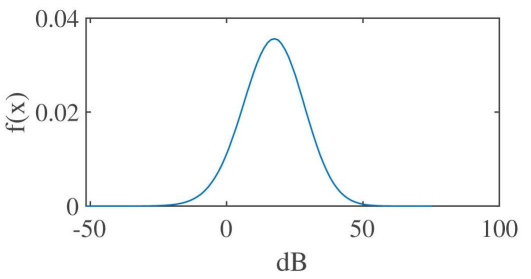

(c)

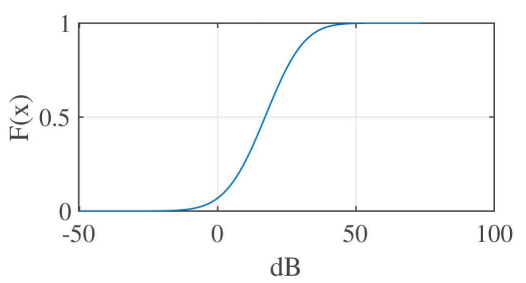

(d)

Figure 2. Rayleigh channel modeling statistics. (a) SINR statistics Rayleigh; (b) Histogram; (c) Estimated PDF; (d) Empirical CDF. 


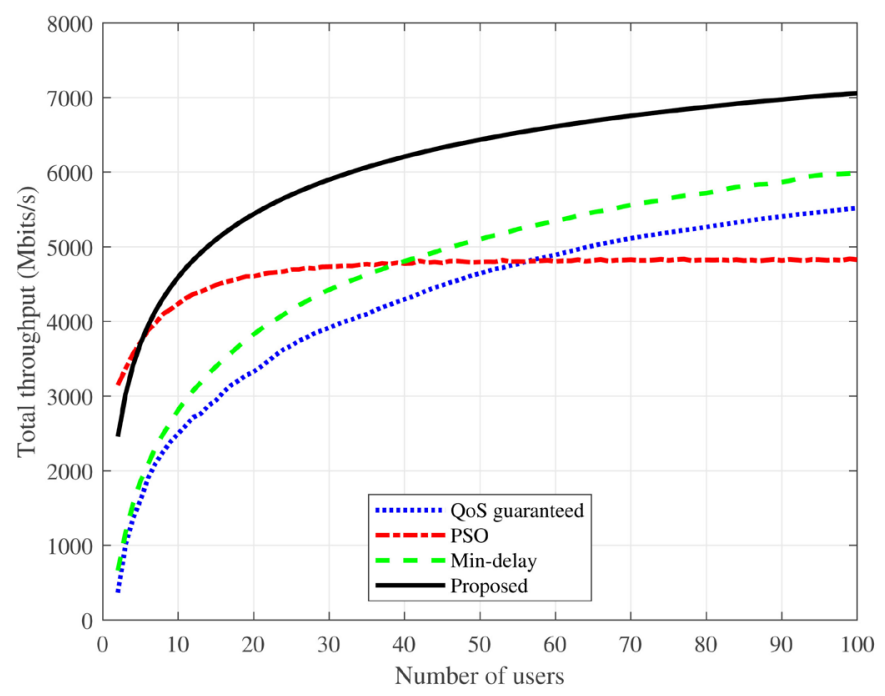

Figure 3. Total throughput for TDL-A channel modeling.

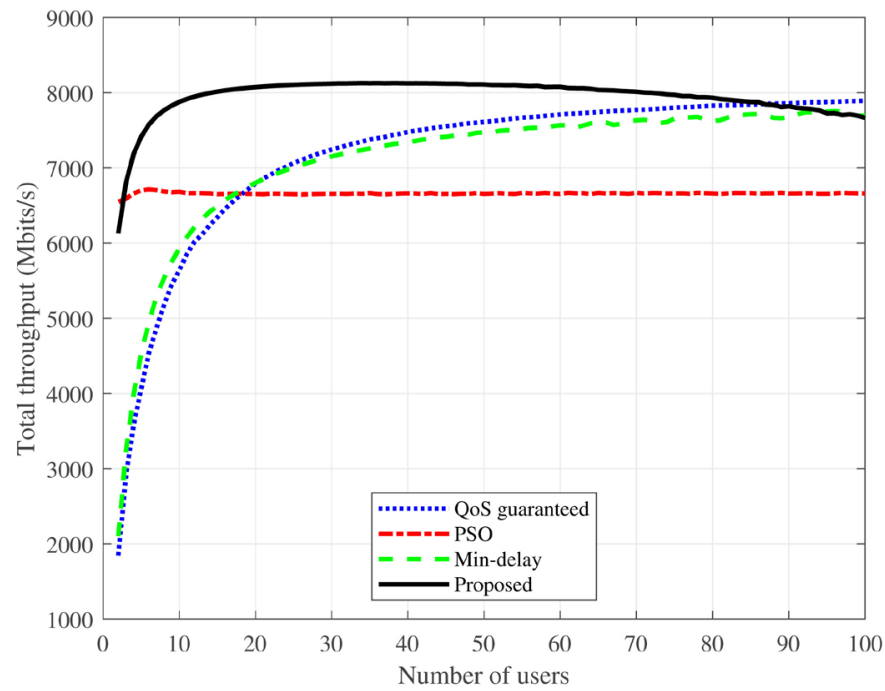

Figure 4. Total throughput for Rayleigh channel modeling.

adequately optimizes the system delay and throughput parameters by using the prediction of the idle states. Once TDL-A modeling represents more severe transmission channel conditions, the throughput values presented by the resource allocation algorithms are lower for this modeling than those with Rayleigh channel modeling. In terms of the average throughput, Figure 5 and Figure 6 show that the proposed algorithm presents the best performance of all the scenarios.

The proposed algorithm presents the lowest delay values for the simulated scenarios, even varying the numbers of users, as shown in Figure 7 and Figure 8. This proves that Algorithm 1 is effective in its proposal of minimizing delay, despite the conditions of the transmission channel.

Figure 9 and Figure 10 reveal that the proposed algorithm provides the lowest delay and highest throughput values at the cost of degradation in the fairness 


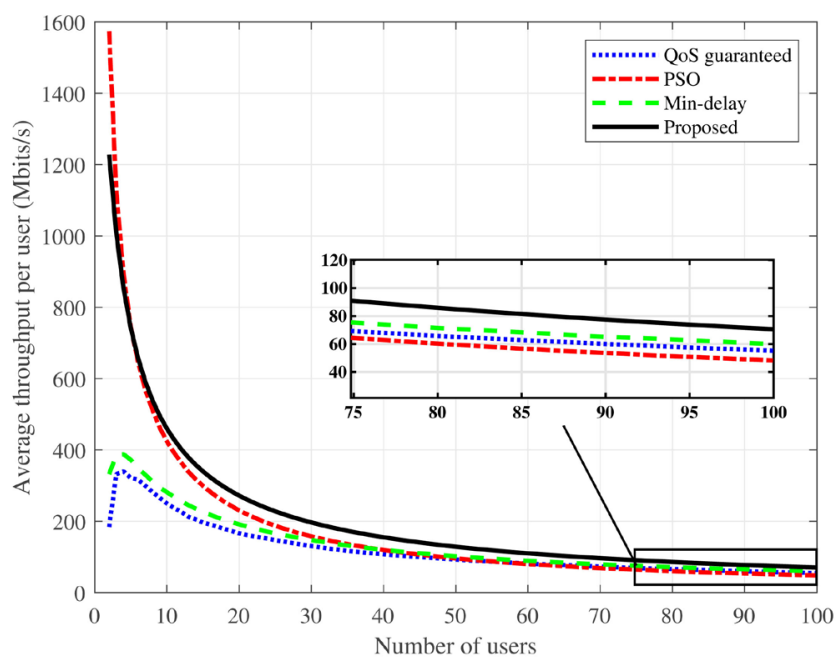

Figure 5. Average throughput for TDL-A channel modeling.

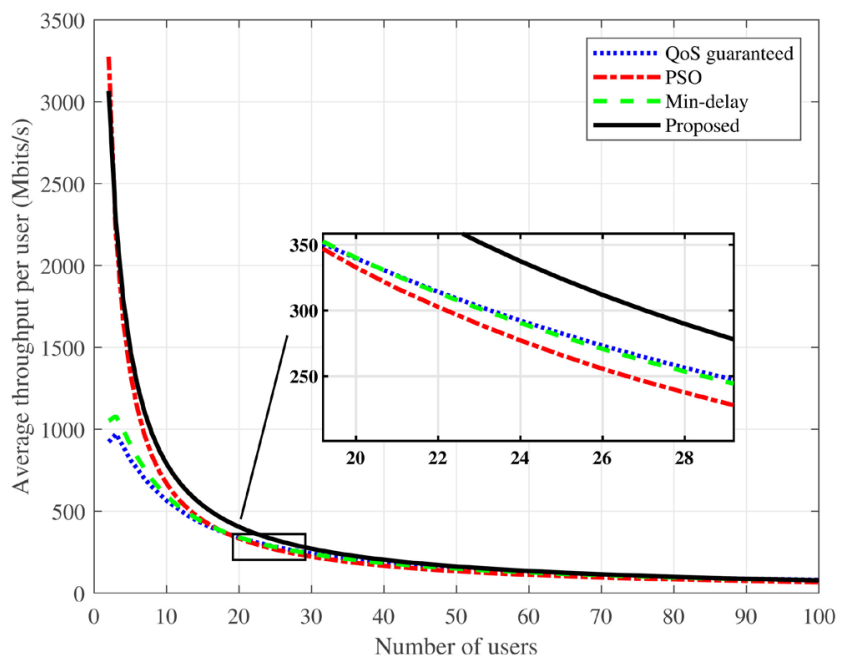

Figure 6. Average throughput for Rayleigh channel modeling.

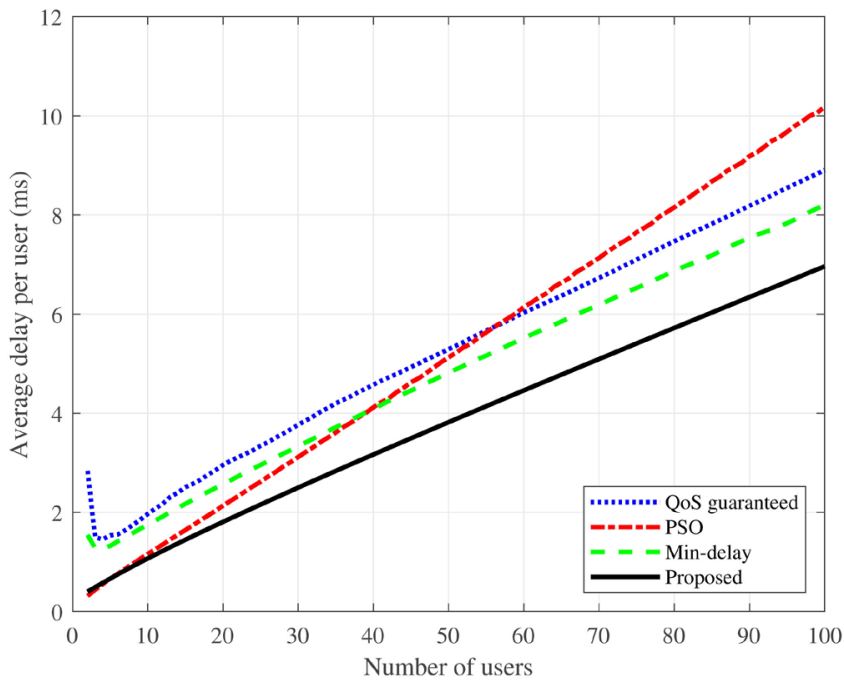

Figure 7. Average delay for TDL-A channel modeling. 


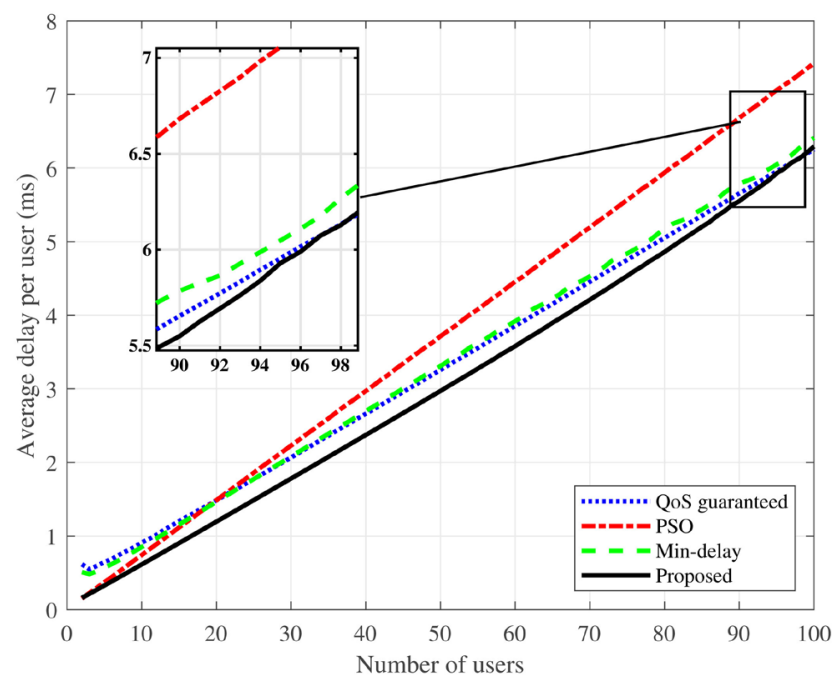

Figure 8. Average delay for Rayleigh channel modeling.

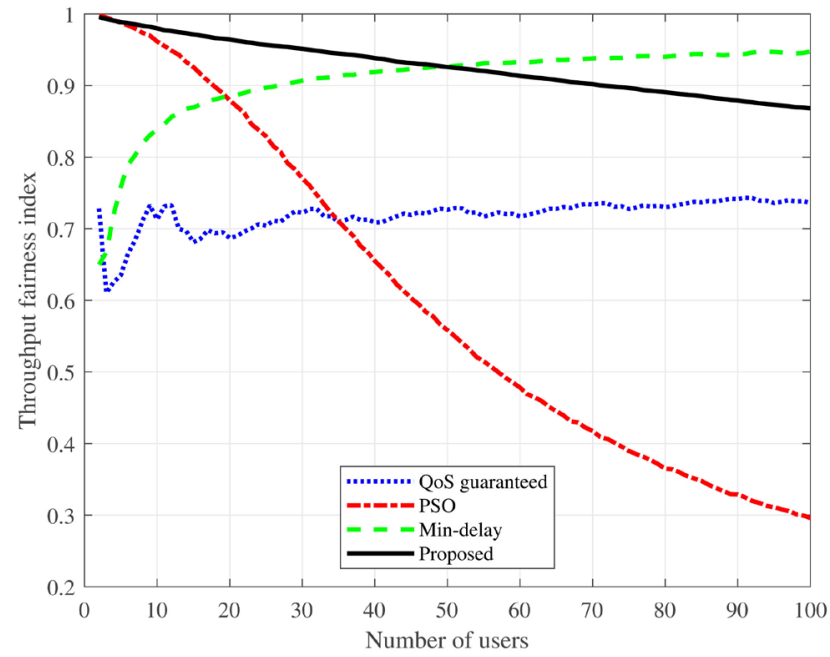

Figure 9. Fairness index for TDL-A channel modeling.

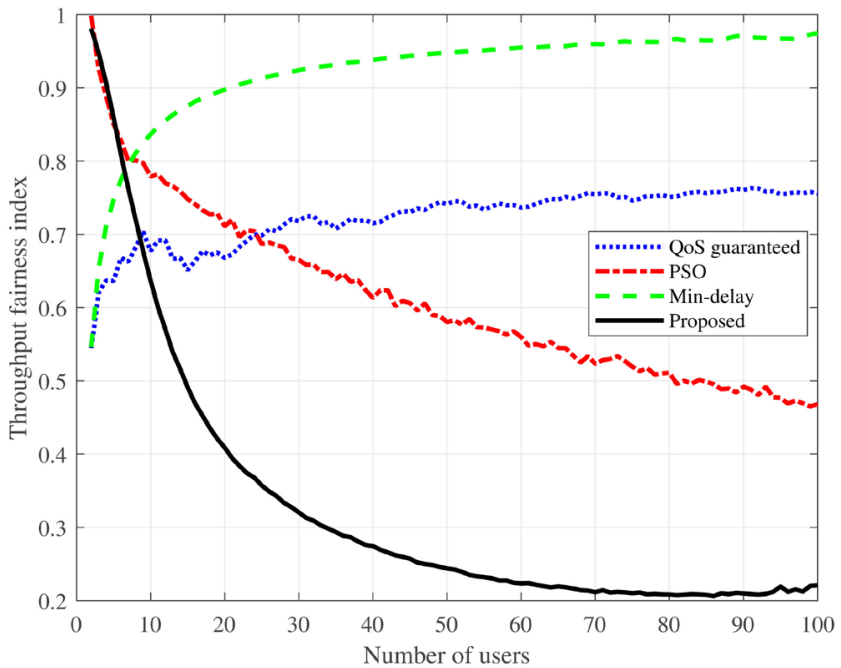

Figure 10. Fairness index for Rayleigh channel modeling. 
values. However, the fairness degradation to the proposed resource allocation algorithm is lower when considering TDL-A modeling than for Rayleigh modeling.

In relation to the data of Table 2, it refers to the percentage mean bit loss in the considered wireless system. It was considered the average of 1000 simulation runs. Analyzing this parameter, it can be noted that the proposed algorithm presents values approaching zero. The Min-delay and QoS guaranteed algorithms also present low loss rate values, which were influenced by the scenarios with high bandwidth and availability of resource blocks. On the other hand, the PSO algorithm presents the worst performance in terms of the loss rate, tending to provide higher loss rate as the number of users is increased. We believe that this behavior of the PSO algorithm is due to the combination of its lower throughput and fairness values in general than the other approaches.

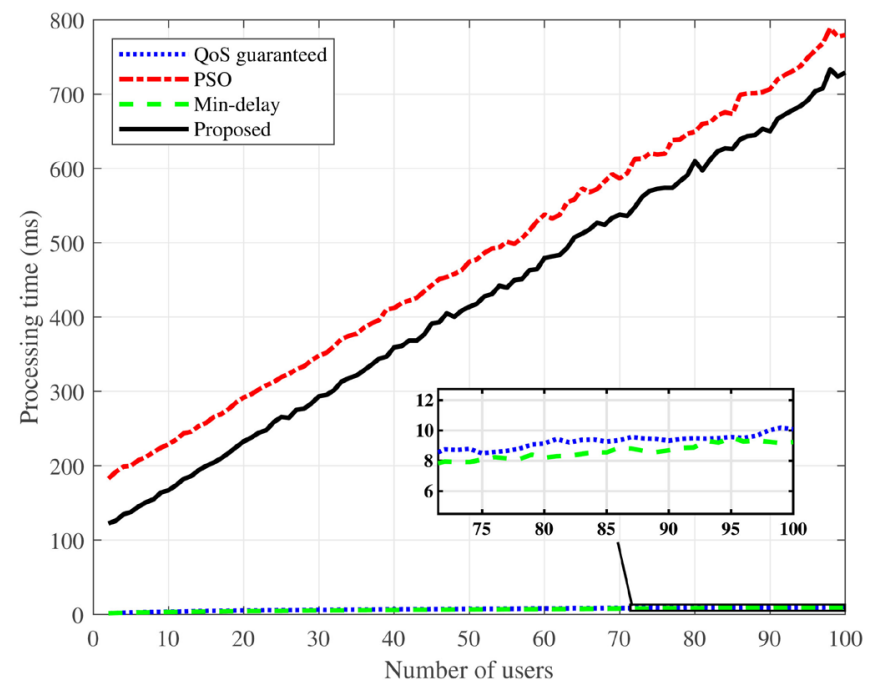

Figure 11. Processing time for TDL-A channel modeling.

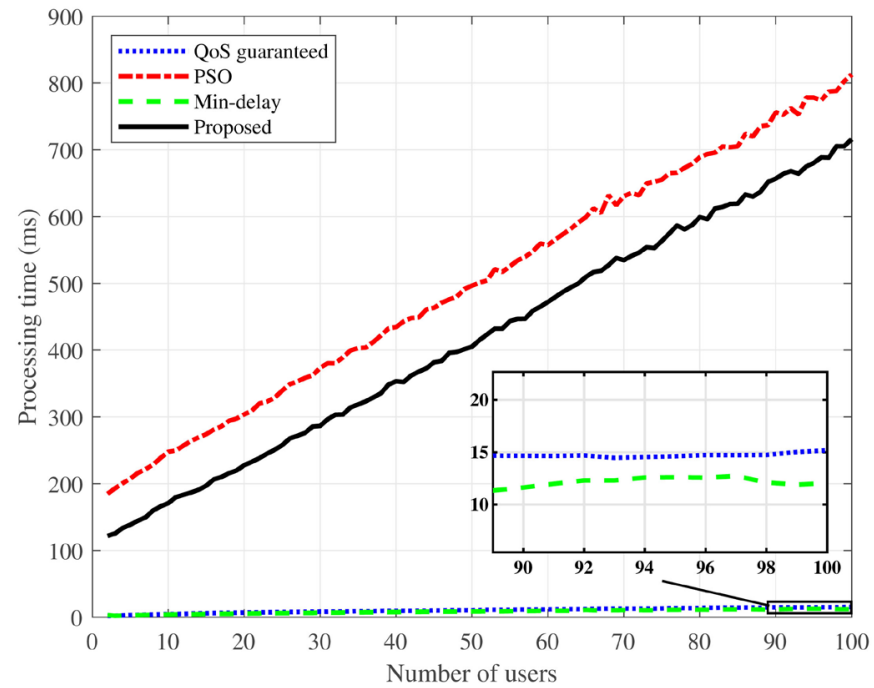

Figure 12. Processing time for Rayleigh channel modeling. 
Table 2. Percentage loss rate (\%) for TDL-A and Rayleigh channel modeling.

\begin{tabular}{ccccccc}
\hline \multirow{3}{*}{ Channel modeling } & Algorithm & \multicolumn{5}{c}{ Number of users } \\
\cline { 3 - 7 } & QoS guaranteed & 0 & 0 & 0.001 & 0.001 & 0.001 \\
\hline \multirow{3}{*}{ TDL-A } & PSO & 0 & 0 & 0 & 0.025 & 0.166 \\
& Min-delay & 0 & 0 & 0.001 & 0.001 & 0.001 \\
& Proposed & 0 & 0 & 0 & 0.001 & 0.001 \\
\hline \multirow{3}{*}{ Rayleigh } & QoS guaranteed & 0 & 0 & 0.001 & 0.001 & 0.001 \\
& PSO & 0 & 0 & 0 & 0.004 & 0.073 \\
& Min-delay & 0 & 0 & 0.001 & 0.001 & 0.001 \\
& Proposed & 0 & 0 & 0 & 0.001 & 0.001 \\
\hline
\end{tabular}

The complexity of the QoS guaranteed and Min-delay algorithms are given in function of the number of block allocation executions subject to the number of users $K$ and blocks $N$, that is, the complexity is $\leq O(N \times K)$. In the analysis of the complexity of the PSO-based scheduler, as being an heuristic algorithm, the number of iterations maxit and the size of the population $P_{o}$ must also be considered. Thus, the computational complexity of the PSO-based scheduler is equal to $O\left(N \times K \times P_{o} \times\right.$ maxit $)$ [14]. In the complexity of the proposed algorithm, the number of executions of the optimization step $u$ to find the optimal $\eta^{*}$ of the idle state prediction algorithm must be included. Thus, its computational complexity is equal to $O(N \times K \times u)$, that is slightly higher than those of the QoS guaranteed and Min-delay algorithms, but lower than that of the PSO-based scheduler, as can be seen from the processing time shown in Figure 11 and Figure 12 .

\section{Conclusions}

This paper proposed a resource block allocation scheme for mmWave wireless networks using current and next-generation techniques, aiming at optimizing the user data delay and maintaining high throughput levels. It was demonstrated that the scheduling policy can be formulated as a total throughput maximization problem and that the choice of weights assigned for computing user rates impacts the delay optimization performance.

Another contribution of the present study consists of evaluating the resource allocation algorithm for a scenario with subcarrier aggregation and mmWave propagation, comparing its performance with other algorithms in the literature and with Rayleigh channel modeling. In fact, the proposed resource allocation algorithm demands lower processing capacity in relation to an algorithm based on particle swarm but with better overall performance, as observed in the simulations.

According to the results, the proposed resource allocation algorithm with delay optimization presented a better performance in terms of delay for all simu- 
lated scenarios with different numbers of users when compared to the PSO, QoS guaranteed and Min-delay algorithms. The proposed algorithm also presents higher total and average throughput values than the other algorithms, loss rate close to zero and suitable fairness values. These results prove that the proposed algorithm is efficient in its proposal of delay optimization, despite the transmission channel conditions.

\section{Acknowledgements}

The authors would like to thank Fundação de Amparo à Pesquisa no Estado de Goiás (FAPEG) for their support in the development of the research.

\section{Conflicts of Interest}

The authors declare no conflicts of interest regarding the publication of this paper.

\section{References}

[1] 3GPP (2017) 3GPP TR 38.901 Version 14.0.0 Release 14. 5G; Study on Channel Model for Frequencies from 0.5 to $100 \mathrm{GHz}$.

[2] 3GPP (2018) 3GPP TS 38.211 Version 15.2.0 Release 15.5G; NR; Physical Channels and Modulation.

[3] Zaidi, A. A., Baldemair, R., Tullberg, H., Bjorkegren, H., Sundstrom, L., Medbo, J., Kilinc, C. and Da Silva, I. (2016) Waveform and Numerology to Support 5G Services and Requirements. IEEE Communications Magazine, 54, 90-98. https://doi.org/10.1109/MCOM.2016.1600336CM

[4] Rostami, S., Arshad, K. and Rapajic, P. (2015) A Joint Resource Allocation and Link Adaptation Algorithm with Carrier Aggregation for 5G LTE-Advanced Network. 2015 22nd International Conference on Telecommunications (ICT), Sydney, 27-29 April 2015, 102-106. https://doi.org/10.1109/ICT.2015.7124665

[5] Ferreira, M.V.G., Vieira, F.H.T. and Abrahao, D.C. (2015) Minimizing Delay in Resource Block Allocation Algorithm of LTE Downlink. 2015 International Workshop on Telecommunications (IWT), Santa Rita do Sapucai, 14-17 June 2015, 1-7. https://doi.org/10.1109/IWT.2015.7224550

[6] Guan, N., Zhou, Y., Tian, L., Sun, G. and Shi, J. (2011) QoS Guaranteed Resource Block Allocation Algorithm for LTE Systems. 2011 IEEE 7 th International Conference on Wireless and Mobile Computing, Networking and Communications (WiMob), Wuhan, 10-12 October 2011, 307-312. https://doi.org/10.1109/WiMOB.2011.6085383

[7] Su, L., Wang, P. and Liu, F. (2012) Particle Swarm Optimization Based Resource Block Allocation Algorithm for Downlink LTE Systems. 201218 th Asia-Pacific Conference on Communications (APCC), Jeju Island, Korea, 15-17 October 2012, 970-974.

[8] Gupta, A. and Jha, R.K. (2015) A Survey of 5G Network: Architecture and Emerging Technologies. IEEE Access, 3, 1206-1232. https://doi.org/10.1109/ACCESS.2015.2461602

[9] 3GPP (2016) 3GPP TS 36.213 Version 13.0.0 Release 13. LTE; Evolved Universal Terrestrial Radio Access (E-Utra); Physical Layer Procedures. 
[10] 3GPP (2018) 3GPP TS 38.104 Version 15.2.0 Release 15. 5G; NR; Base Station (BS) Radio Transmission and Reception.

[11] Zhou C. and Wunder, G. (2007) Delay and Throughput Optimal Scheduling for OFDM Broadcast Channels. Proc. IEEE Globecom 2007. IEEE International Symposium on Information Theory (ISIT2007), Nice, France, 24-29 June 2007.

[12] Ericsson (2019) In the Race to 5G, CP-OFDM Triumphs. In-the-Race-to-5G-CPOFDM-Triumphs, May 2019, 27. https://www.ericsson.com/en/blog/2017/5/

[13] 3GPP (2019) 3GPP TS 38.306 Version 15.7.0 Release 15. 5G; NR; User Equipment (UE) Radio Access Capabilities.

[14] Abrahão, D.C. (2018) Escalonamento de recursos em redes LTE utilizando processo envelope de tráfego multifractal e curva de serviço mínima. PhD Thesis, Escola de Engenharia Eletrica, Mecânica e de Computação-EMC (RG). 